\title{
Uso de Plantas Medicinais e Fitoterápicos na Atenção Primária à Saúde: uma revisão integrativa
}

\author{
Use of Medicinal and Phytotherapeutic Plants in Primary Health Care: an integrative \\ review
}
Uso de Plantas Medicinales y Fitoterápicos en la Atención Primaria a la Salud: una revisión integrativa

Igor de Moura Costa ${ }^{1}$, Isnayara da Rocha de Alencar ${ }^{1}$, Juliana Anchieta de Lyra ${ }^{1}$, Marcus Francisco Sobrinho do Nascimento ${ }^{1}$, Raimundo Nonato da Silva Júnior ${ }^{1}$, Stanley Sousa Damasceno ${ }^{1}$, Ivana Cavalcante Lemos de Sousa ${ }^{1 *}$, Daniel de Macedo Rocha ${ }^{2}$.

\section{RESUMO}

Objetivos: analisar uso de plantas medicinais e suas aplicações na cura e prevenção de doenças e verificar a prática da fitoterapia na atenção primária à saúde. Métodos: trata-se de uma revisão bibliográfica de literatura pelo método revisão integrativa, qualitativa e exploratória por meio das publicações selecionadas nas bases de dados online. A análise dos dados foi realizada por meio da leitura detalhada do conteúdo dos artigos categorizados por similaridade/semântica. Resultados: Após o levantamento, utilizando como critério de escolha os estudos publicados nos anos de 2014 a 2019, obteve-se 12 artigos relevantes ao tema, com prevalência nos idiomas português e inglês e publicados no ano de 2017. Conclusão: a fitoterapia como Prática Integrativa Complementar tem se expandido na APS por ser uma medida vantajosa que causa menos efeitos colaterais, menor dependência, menor custo e maior facilidade de acesso para a população. Para a sua efetividade dentro da APS tornasse necessário habilitar os profissionais de saúde para a prescrição dos fitoterápicos, com base em conhecimentos técnico-científicos além de realizar capacitação da comunidade que irá utilizá-la.

Palavras-chave: Fitoterapia, Plantas Medicinais, Atenção Primária à Saúde.

\begin{abstract}
Objectives: the use of medicinal plants and their applications in curing and preventing diseases and in primary health care therapy. Methods: This is a bibliographical review of the literature using the integrative, qualitative and exploratory review method for publications in online databases. Data analysis was performed by means of a detailed reading of articles categorized by similarity / semantics. Results: After publication, it was used as a study option the results from the years 2014 to 2019, was published on June 12, 2017. Conclusion: phytotherapy as Complementary Integrative Practice expanded in PHC because it was an advantageous measure that justified the influence of the side effects, less dependence, lower cost and greater ease of access for the population. To achieve its effectiveness under the PHC, it is necessary to enable health professionals to select herbal medicines, based on technical-scientific criteria, in addition to training the community that will use it.
\end{abstract}

Key words: Phytotherapy, Medicinal Plants, Primary Health Care.

${ }^{1}$ Centro Universitário Santo Agostinho (UNIFSA), Teresina-PI.*E-mail: ivana_cls@hotmail.com

2 Universidade Estadual do Piauí (UESPI), Teresina-PI. 


\section{RESUMEN}

Objetivos: uso de plantas medicinales y sus aplicaciones en la cura y en la prevención de enfermedades y en la terapia de la atención primaria a la salud. Métodos: se trata de una revisión bibliográfica de literatura por el método de revisión integrativa, cualitativa y exploratoria para las publicaciones en las bases de datos online. El análisis de los datos fue realizado por medio de la lectura detallada de los artículos categorizados por similitud / semántica. Resultados: Después de la publicación, utilizó como opción de estudios los resultados de los años 2014 a 2019, fue publicado el 12 de junio de 2017. Conclusión: la fitoterapia como Práctica Integradora Complementaria se expandió en la APS por ser una medida ventajosa que justificó la influencia de los efectos colaterales, menor dependencia, menor costo y mayor facilidad de acceso para la población. Para obtener su eficacia en el ámbito de la APS, es necesario habilitar a los profesionales de salud para una selección de los fitoterápicos, con base en criterios técnico-científicos, además de realizar la capacitación de la comunidad que la utilizará.

Palabras clave: Fitoterapia, Plantas Medicinales, Atención Primaria a la Salud.

\section{INTRODUÇÃO}

O aumento da procura por práticas terapêuticas que se integram e complementam a prática clínica na saúde e sua progressiva aceitação por profissionais da área é fato relativamente recente, em virtude do reconhecimento de relevância social, acadêmica e profissional, que corroboram a percepção de que o cuidado direcionado ao indivíduo vai além da dimensão clínica que em sua maioria remete a medicalização, mais envolve um pluralismo terapêutico (CASTRO SST e TESSER CD, 2011).

Nesse contexto de multiplicidade terapêutica no âmbito do Sistema Único de Saúde (SUS) destacam-se, as práticas Integrativas e complementares das quais a fitoterapia é um dos eixos mais importantes, que se introduz no cuidado através da Atenção Primária à Saúde. A Fitoterapia é caracterizada pelo uso de plantas medicinais em suas diferentes formas farmacêuticas. $O$ uso de plantas medicinais na arte de curar é uma forma de tratamento de origens muito antigas, relacionada aos primórdios da medicina e fundamentada no acúmulo de informações por sucessivas gerações. Ao longo dos séculos, produtos de origem vegetal constituíram as bases para tratamento de diferentes doenças (BRASIL, 2006).

A Atenção Primária à Saúde - APS se constitui como campo imprescindível para a efetivação da Fitoterapia enquanto modalidade terapêutica associada ao tratamento medicamentoso e até mesmo visando a prevenção, haja vista que, a comunidade e os usuários organizados, com suas tradições, valores e saberes, bem como as instituições acadêmicas, de pesquisa e ensino, com seus critérios científicos, profissionais da saúde podem contribuir na construção de uma ecologia de saberes sobre plantas medicinais, em diálogos e decisões sobre os usos/orientações/prescrições das plantas medicinais e fitoterápicas na APS, envolvendo o saber científico, o popular e o cultural (ROSA CD et al., 2011).

Dessa forma a fitoterapia na APS segundo Fontenele RP et al. (2013), visa a prevenção de agravos, a promoção e a recuperação da saúde com ênfase na atenção básica. Além disso, propõe o cuidado continuado da saúde, afim contribuir para o aumento da resolutividade do sistema com qualidade, eficiência, segurança, sustentabilidade, controle e participação social, respeitando os princípios do SUS, sobretudo a integralidade da assistência, e resguardando o direito fundamental e inalienável a saúde.

A partir disso, definiu-se como objetivos analisar uso de plantas medicinais e suas aplicações na cura e prevenção de doenças, como também verificar a utilização de plantas medicinais na prática da fitoterapia na atenção primária à saúde.

\section{MÉTODOS}

O presente trabalho trata de uma revisão bibliográfica de literatura pelo método revisão integrativa, qualitativa e exploratória por meio das publicações selecionadas nas bases de dados online. 
A busca do material ocorreu por meio da estratégia PICo ( $\mathrm{P}=$ população, paciente ou problema; I=Interesse; $\mathrm{Co}=$ Contexto), por busca avançada, sendo o Problema, fitoterapia, o Interesse foi a utilização das plantas medicinais na fitoterapia, e o Contexto foi a utilização das plantas medicinais na fitoterapia na atenção primária à saúde. Os dados contidos nos artigos selecionados foram levantados pelos pesquisadores por meio de um formulário organizado em dois eixos, sendo o primeiro, que trata das características das publicações, e o segundo, que trata dos resultados e das evidências neles contidos. A busca de dados foi realizada por meio dos descritores: Fitoterapia; Plantas Medicinais; Atenção Primária à Saúde. Sendo a base de dados utilizada: Biblioteca Virtual em Saúde - BVS.

Para seleção dos artigos foram utilizados os seguintes critérios de inclusão: artigos originais, completos e disponíveis na íntegra, que estejam no idioma português e inglês, publicados nos últimos 6 anos e que sejam relacionados com o tema. Já como critério de exclusão optou-se por não utilizar artigos que não sejam relacionados ao tema, dissertação de mestrado e tese de doutorado, artigos que estejam em outros idiomas e fora do período pré-estabelecido.

A análise dos dados foi realizada por meio da leitura detalhada do conteúdo dos artigos categorizados por similaridade/semântica. Os resultados foram apresentados em categorias intituladas de acordo com 0 conteúdo e discutidos à luz do referencial. A seleção foi realizada em duplo cego por meio dos pesquisadores da equipe de revisão. Também foi realizado o cruzamento dos descritores utilizando o operador booleano AND, para uma pesquisa precisa acerca do tema.

\section{RESULTADOS}

Durante a elaboração da pesquisa levantou-se 72 estudos. Após este levantamento, utilizou-se como critério de escolha os estudos publicados nos anos de 2014 a 2019, com prevalência em idiomas em português, com textos completos e que fossem relevantes ao tema. Com isso obteve-se 12 publicações refinadas de acordo com os objetivos do estudo e critérios de inclusão e exclusão, distribuídas em diferentes periódicos (Tabela 1).

Tabela 1 - Distribuição das publicações conforme ano e título do periódico. Teresina, 2019.

\begin{tabular}{llll}
\hline Ano & Periódico & Frequência & $\%$ \\
\hline 2014 & Revista de Atenção Primária em Saúde & 01 & 8,33 \\
2014 & Revista de Saúde Pública & 01 & 8,33 \\
2015 & Revista Brasileira de Medicina de Família e Comunitária & 01 & 8,33 \\
2015 & Journal of Research Fundamental Care Online & 02 & 16,67 \\
2016 & Revista de Ciência em Extensão & 01 & 8,33 \\
2017 & Revista Fitos & 01 & 8,33 \\
2017 & Journal of Research Fundamental Care Online & 01 & 8,33 \\
2017 & Revista Ciência \& Saúde Coletiva & 02 & 16,67 \\
2018 & Revista Ciência \& Saúde Coletiva & 01 & 8,33 \\
2018 & Boletín Latinoamericano Y del Caribe de Plantas Medicinales Y Aromáticos & 01 & 8,33
\end{tabular}

Fonte: Biblioteca Virtual em Saúde - BVS.

Na realização do cruzamento dos descritores e após a filtragem ficaram apenas 12 (16,68\%) estudos para o corpus da pesquisa. Com relação ao idioma, 7 artigos encontravam-se em português e 6 em inglês. De acordo com o levantamento realizado na base de dados da BVS, contemplaram-se as produções mais atualizadas acerca da Utilização de Plantas Medicinais em Fitoterapia na Atenção Primária à Saúde (Quadro 1). 
Quadro 1 - Distribuição das referências incluídas na revisão integrativa, de acordo com as bases de dados, idioma e tipo de estudo. Teresina, 2019.

\begin{tabular}{|l|l|l|}
\hline Base de dados & Idioma & Tipo de estudo \\
\hline BVS & Português & Qualitativa/Quantitativa \\
\hline BVS & Português & Quantitativa \\
\hline BVS & Português & Quantitativa \\
\hline BVS & Português & Qualitativa \\
\hline BVS & Português & Qualitativa \\
\hline BVS & Português & Quantitativa \\
\hline BVS & Português & Qualitativa \\
\hline BVS & Português & Quantitativa \\
\hline BVS & Inglês & Qualitativa \\
\hline BVS & Inglês & Qualitativa \\
\hline BVS & Inglês & Qualitativa \\
\hline BVS & Inglês & Quantitativa \\
\hline
\end{tabular}

Fonte: Biblioteca Virtual em Saúde - BVS.

Através da análise dos dados coletados, foram encontrados artigos com prevalência no ano de 2017, com 4 estudos (33,33\%), encontrou-se 3 estudos no ano de 2015 (24,99\%), 2 no ano de 2014 (16,66\%), 2 no ano de 2018 (16,66\%) e 1 no ano de 2016 (8,33\%), não houve estudos relacionados à temática no ano de 2019. Isso possibilita a busca por maior conhecimento acerca do tema da pesquisa, no intuito de atualização profissional.

\section{DISCUSSÃO}

Diante das apresentações das publicações o estudo permitiu fazer uma análise de duas categorias temáticas que estão descritas abaixo:

\section{Uso de Plantas Medicinais e suas Aplicações na Cura e Prevenção de Doenças e Alívio de Sinais e Sintomas}

No Brasil, o uso de plantas medicinais em algumas comunidades e grupos étnicos mais isolados ainda é a única forma terapêutica de tratar doenças transmissíveis, e com isso o interesse popular nesta temática é cultural. Ainda hoje é possível encontrar a comercialização de produtos de origem vegetal, que na maioria das vezes sofrem associações para potencializar o efeito desejado (SANTOS JAA et al., 2016).

Ainda segundo Santos JAA et al. (2016), o uso de plantas medicinais está relacionado à cultura popular que é transmitida ao longo de gerações, nas comunidades tradicionais e também nas populações contemporâneas através do conhecimento empírico, que constitui um importante campo gerador do conhecimento etnobotânico, o qual permite avaliar a relação entre o homem e as plantas medicinais, permitindo a descoberta de compostos com ações farmacológicas e aplicações terapêuticas, compartilhando os saberes populares e científicos.

De acordo com Palma JS et al. (2015), o uso de plantas no processo saúde-doença sempre foi questionado por muitos, porém, seu uso foi por anos a única opção para resolver necessidades básicas, consistindo de registros e manuscritos de diferentes civilizações. Enquanto a percepção de saúde e doença aproximava-se do caráter místico e religioso, o uso das plantas foi menos denegrido. Na medida em que a ciência se desenvolveu, as críticas aumentaram consideravelmente. Várias plantas foram a base para a produção de medicamentos alopáticos, como agentes antimicrobianos, e substituíram temporariamente o uso dessas plantas industriais por drogas durante o século XX. No entanto, a alta toxicidade e o alto custo de produção despertaram novamente o interesse pelas plantas, devido aos menores custos e tempo de produção.

Ademais, a fitoterapia consiste em um elo de terapias complementares, que são técnicas voltadas à atenção à saúde do indivíduo, seja na prevenção, tratamento ou cura, considerando o homem como um todo, 
em vez de um conjunto de partes isoladas. Com o desenvolvimento da indústria farmacêutica e da mudança de paradigma na construção de conhecimento em saúde, este uso tem diminuído, mas nas últimas décadas, terapias complementares se expandiram em todo o mundo, o que pode estar relacionado com o alto preço dos cuidados médicos privados, alto custo dos medicamentos fabricados, precariedade assistência e tradição do uso de plantas como suplemento terapêutico. Além disso, a necessidade da população de controlar seu próprio corpo e restaurar saúde, adotando práticas de saúde para si e para a família, associados aos perigos do uso abusivo e irracional de produtos farmacêuticos está levando a um número maior de práticas complementares e integrativas, dentre as principais, está a fitoterapia (ARAÚJO AKL et al., 2015)

Atualmente, a maior parte da população depende do uso de plantas medicinais nos cuidados de saúde. No entanto, a medicina moderna está lado a lado com a prática da fitoterapia tradicional em manter seu uso por razões e fatores culturais, onde contribuem positivamente com o processo saúde-doença, seja o uso de ervas como chás, puros e liquidificados ou mesmo os fitoterápicos, sempre no intuito de diminuir a dependência do uso de medicamentos alopáticos (GRIBNER C et al., 2018).

Quadro 2 - Resumo das conclusões de estudo sobre o Uso de Plantas Medicinais e suas Aplicações na Cura e Prevenção de Doenças e Alívio de Sinais e Sintomas. Teresina, 2019.

\begin{tabular}{|c|l|}
\hline Estudos & \multicolumn{1}{|c|}{ Conclusões Importantes } \\
\hline SANTOS JAA et al. (2016) & $\begin{array}{l}\text { É possível considerar o uso consciente e seguro das plantas em } \\
\text { tratamentos de enfermidades de baixa e média complexidade, bem como } \\
\text { ampliando a busca pelo conhecimento sobre as ações farmacológicas e } \\
\text { segurança no uso de diversas espécies. }\end{array}$ \\
\hline PALMA JS et al. (2015) & $\begin{array}{l}\text { Para o autor, o executante deve buscar investir na sensibilização para } \\
\text { discussões frutíferas sobre a implementação de programas locais de } \\
\text { saúde, voltados para o cultivo, manejo e distribuição de plantas medicinais } \\
\text { na atenção primária à saúde. }\end{array}$ \\
\hline ARAÚJO AKL et al. (2015) & $\begin{array}{l}\text { É essencial que os integrantes da equipe da Estratégia Saúde da Família } \\
\text { desenvolvam estratégias efetivas para auxiliar no enfrentamento das } \\
\text { dificuldades enfrentadas, uma vez que essas terapias são consideradas } \\
\text { muito importantes na vida dos usuários e precisam ser praticadas. }\end{array}$ \\
\hline GRIBNER C et al. (2018) & $\begin{array}{l}\text { Foi evidenciado uma boa aceitação dos medicamentos fitoterápicos } \\
\text { industrializados pelos pacientes durante os tratamentos, uma vez que } \\
\text { esses tratamentos demonstraram eficácia e segurança, de acordo com os } \\
\text { usuários entrevistados. }\end{array}$ \\
\hline
\end{tabular}

Fonte: Dados da Pesquisa, 2019.

\section{A Prática da Fitoterapia na Atenção Primária à Saúde - APS}

Para que haja possibilidade de fazer uso da Fitoterapia no âmbito do SUS é necessário que se tenha conhecimento sobre cada planta medicinal, quais efeitos causam, desde benéficos à maléficos. De acordo com Colet CR et al. (2015) o profissional que utiliza essa prática deve obrigatoriamente relacionar o conhecimento empírico com o científico, para utilização correta e segura das plantas, pois o uso incorreto pode ocasionar efeitos adversos, como o cultivo inadequado, identificação incorreta da farmacobotânica, informações insuficientes sobre os efeitos adversos como uma possível reação alérgica, esquema posológico, tempo de uso da terapia e interações medicamentosas.

Ainda de acordo com Colet CR et al. (2015), é importante também identificar os usuários dessa terapia e capacitá-los quanto a utilização das plantas medicinais e fitoterápicos. Assim, torna-se necessário a aplicação de estudos epidemiológicos sobre o uso das mesmas, para identificação de doenças passíveis deste tratamento e criação de programas educacionais que ofereçam informações sobre sua eficácia e segurança. 
A demanda para a implementação de fitoterapia no SUS resultou na criação de uma política nacional de saúde. Em 2006, foi aprovada a Política Nacional de Práticas Integrativas e Complementares (PNPIC) pela Portaria do Ministério da Saúde no 971 de 3 de maio de 2006 e a Política Nacional de Plantas Medicinais e Fitoterápicos (PNPMF), através do Decreto ํo 5813 de 22 de junho de 2006. No Brasil, o incentivo à formação de Arranjo Produtivo Legal (APL) é uma estratégia do governo para o desenvolvimento do país. Essa medida resultou ao longo dos anos na estruturação de um grande número de APL's em diversos segmentos econômicos e industriais, como os setores de madeira e móveis, cerâmica, couro e calçados, metalúrgica, turismo, agricultura, entre outros. Nesse intuito, o Ministério da Saúde utiliza diretrizes da Política Nacional de Plantas Medicinais e Fitoterápicos (PNPMF) para publicar editais para seleção de projetos de APL em plantas medicinais e fitoterápicos no âmbito do SUS (FERREIRA LLC et al., 2017).

Para Oliveira AFP et al. (2017) é necessário a criação de políticas públicas para aumentar o uso de terapias alternativas, como a fitoterapia, que visa reduzir a disparidade em relação à manutenção e melhoria da saúde na população, devido à grande desigualdade social, onde predomina falta de acesso a médicos e tratamentos medicamentosos por esses usuários. A partir disso, a inserção da prática da fitoterapia em unidades de APS tem sido encorajada, já que estudos mostram que $80 \%$ da população faz uso de plantas medicinais e suas preparações.

Na pesquisa de Varela DSS e Azevedo DM (2014), onde foram entrevistados médicos e enfermeiros da APS, os autores citam como mais vantajosa a utilização de plantas medicinais e fitoterápicos, pois provocam menos efeitos colaterais e adversos do que os medicamentos alopáticos, além de causar menor dependência ou nenhuma. Os entrevistados ressaltaram também que a associação da planta medicinal ou do fitoterápico à terapia alopática tem se mostrado uma boa alternativa para tratamento de algumas doenças, pois diminui a quantidade de medicamentos alopáticos usados ou sua dosagem.

Após todas as vantagens apresentadas acima, a principal delas é o baixo custo e o fácil acesso, podendo ser facilmente implantada na comunidade como alternativa para tratamentos de várias doenças, assim, diminuindo a necessidade de compra e distribuição de analgésicos, anti-inflamatórios, psicotrópicos, entre outros. A implantação de hortas com plantas medicinais nas unidades de atenção primária à saúde ou programas de acompanhamento da plantação das mesmas na comunidade é uma boa alternativa para pessoas de baixa renda, que não tem condições financeiras para compra de medicamentos de uso prolongado e também para economia governamental, que distribuirá menos medicamentos, considerando os que tem a mesma finalidade de algumas plantas medicinais (ZENI ALB et al., 2017).

Para Caccia-Brava MCGG et al. (2017), inserir as plantas medicinais e fitoterápicos na nas unidades de atenção primária à saúde pode aumentar a vigilância ao consumo da população pelos profissionais de saúde, sendo sua dispensação na Unidade Básica de Saúde - UBS e sob orientação adequada por um profissional devidamente capacitado. Essa vigilância pode ser ainda mais eficaz se no próprio território da UBS fossem implantadas hortas com o objetivo de obter esses produtos.

O uso da fitoterapia tem motivações diversas, como aumentar os recursos terapêuticos, acima citado, resgatar saberes populares, preservar a biodiversidade, fomentar a agroecologia, desenvolvimento social e a educação ambiental, popular e permanente. Na pesquisa de Antonio GD et al. (2014), foram estudados a aplicação da fitoterapia em algumas unidades de APS, onde foi observado melhora no acesso a outras possibilidades terapêuticas, confirmando todas as vantagens citadas ao longo dessa discussão, em algumas delas, houve até ações de extensão e parcerias com universidades e empresas de agricultura, meio ambiente e educação.

Contudo, pode-se confirmar que a terapia com plantas medicinais é uma via interessante na atenção primária à saúde, visto que uma grande porcentagem de pessoas a preferem em relação a terapia medicamentosa. Na pesquisa de Mattos G et al. (2018), todos os profissionais de saúde entrevistados revelaram o desejo de trabalhar com tal terapia, pois a mesma é geralmente aplicada apenas em unidades PMAQ. Assim, abrem-se portas para incentivo do governo na sua aplicação e à população em geral em relação a exigência para a mesma, visto que só traz benefícios.

REAS/EJCH | Vol. Sup.26 | e828 | DOI: https://doi.org/10.25248/reas.e828.2019 Página 6 de 8 
Quadro 3 - Resumo das conclusões de estudo sobre a prática da Fitoterapia na Atenção Primária à Saúde APS. Teresina, 2019.

\begin{tabular}{|c|c|}
\hline Estudos & Conclusões Importantes \\
\hline COLET CR et al. (2015) & $\begin{array}{l}\text { Para que possa se propagar o conhecimento e se fazer um uso seguro de } \\
\text { plantas medicinais e fitoterápicos, os profissionais da saúde devem estar } \\
\text { habilitados para a prescrição dos mesmos, com base em conhecimentos } \\
\text { técnico-científicos e princípios éticos, considerando as resoluções } \\
\text { profissionais bem como as da Anvisa, buscando, dessa maneira, atender de } \\
\text { forma racional e qualificada às necessidades da população que buscam } \\
\text { atendimento nas Unidades da Atenção Primária à Saúde. }\end{array}$ \\
\hline $\begin{array}{l}\text { FERREIRA LCC et al. } \\
\qquad(2017)\end{array}$ & $\begin{array}{l}\text { Os recursos financeiros obtidos do Ministério da Saúde foram de grande } \\
\text { importância para projetos de APL, visto que possibilitaram às secretarias de } \\
\text { saúde municipais e estaduais a aquisição de materiais e equipamentos para } \\
\text { implantar e/ou adequar programas e ações com plantas medicinais e } \\
\text { fitoterápicos em suas regiões. }\end{array}$ \\
\hline $\begin{array}{l}\text { OLIVEIRA AFP et al. } \\
\qquad(2017)\end{array}$ & $\begin{array}{l}\text { O advento da evidência científica e a implementação das políticas públicas no } \\
\text { SUS, atreladas a práticas antigas que persistem nos dias atuais, revelam e } \\
\text { confirmam a magnitude da Fitoterapia, cujo objetivo principal é promover e } \\
\text { restaurar a saúde da população em geral, mais especificamente, no contexto } \\
\text { da atenção primária. }\end{array}$ \\
\hline $\begin{array}{c}\text { VARELA DSS e } \\
\text { AZEVEDO DM (2014) }\end{array}$ & $\begin{array}{l}\text { Foram mencionados o bom resultado terapêutico, por ocasionarem menores } \\
\text { efeitos colaterais e adversos do que alguns medicamentos alopáticos, a } \\
\text { redução de custos e fácil acesso, devido à riqueza do território nacional, a } \\
\text { união do saber científico à prática popular, por constituir numa forma de } \\
\text { dialogar com a comunidade e aproximá-los de uma prática adequada. }\end{array}$ \\
\hline ZENI ALB et al. (2017) & $\begin{array}{l}\text { Foi possível verificar que a principal forma de uso de remédios caseiros pelos } \\
\text { usuários do SUS foi com plantas medicinais. Além disso, comparando o } \\
\text { número de plantas medicinais citadas pelos usuários neste estudo com outros } \\
\text { similares, com mais usuários deste tipo de terapia, proporcionalmente foi } \\
\text { maior, demonstrando conhecimento popular a ser resgatado. }\end{array}$ \\
\hline $\begin{array}{c}\text { CACCIA-BRAVA MCGG } \\
\text { et al. (2017) }\end{array}$ & $\begin{array}{l}\text { O estudo evidencia também que, no SUS, há um maior uso de fitoterápico } \\
\text { industrializado quando comparado à droga vegetal e ao medicamento } \\
\text { fitoterápico manipulado, o que sinaliza uma necessidade de expansão da } \\
\text { indústria farmacêutica brasileira especializada na produção destes produtos, } \\
\text { para que a crescente utilização deste recurso não fique comprometida por falta } \\
\text { de medicamento no mercado. }\end{array}$ \\
\hline $\begin{array}{c}\text { ANTONIO GD et al. } \\
(2014)\end{array}$ & $\begin{array}{l}\text { Chama a atenção que no país com a maior biodiversidade do mundo, } \\
\text { extensão territorial continental, grande riqueza cultural e de conhecimentos } \\
\text { sobre plantas medicinais, oriunda das três matrizes étnicas formadoras da } \\
\text { população brasileira (indígena, africana e europeia), a atenção primária à } \\
\text { saúde e o sistema único de saúde tenham tão poucas experiências com ações } \\
\text { e uso de plantas medicinais. }\end{array}$ \\
\hline MATTOS G et al. (2018) & $\begin{array}{l}\text { A adoção da prática de fitoterapia ampliaria as opções referentes à prevenção } \\
\text { e tratamento de agravos e doenças que afetam a população, através da } \\
\text { garantia de acesso e uso das plantas medicinais e dos fitoterápicos, com } \\
\text { segurança, eficácia e qualidade, na perspectiva da integralidade da atenção à } \\
\text { saúde. }\end{array}$ \\
\hline
\end{tabular}

Fonte: Dados da Pesquisa, 2019. 


\section{CONCLUSÃO}

A Fitoterapia como Prática Integrativa Complementar tem se expandido na APS por ser uma medida vantajosa que causa menos efeitos colaterais, menor dependência, menor custo e maior facilidade de acesso para a população. Para a sua efetividade dentro da APS torna se necessário habilitar os profissionais de saúde para a prescrição dos fitoterápicos, com base em conhecimentos técnico-científicos além de realizar capacitação da comunidade que irá utilizá-la.

\section{REFERÊNCIAS}

1. ANTONIO GD, et al. Fitoterapia na atenção primária à saúde. Revista de Saúde Pública, 2014; 48(3): $541-553$.

2. ARAÚJO AKL, et al. Difficulties faced by nurses on the applicability of phytotherapy in the basic attention: an integrative review. Journal Research Fundamental Care Online, 2015; 7(3): 2826-2834.

3. CACCIA-BRAVA MCGG, et al. Disponibilidade de medicamentos fitoterápicos e plantas medicinais nas unidades de atenção básica do Estado de São Paulo: resultados do Programa Nacional de Melhoria do Acesso e da Qualidade da Atenção Básica (PMAQ). Revista Ciência \& Saúde Coletiva, 2017; 22(5): 1651-1659.

4. CASTRO SST, TESSER CD. Percepção de Médicos e Enfermeiros da Estratégia de Saúde da Família sobre terapias complementares. Revista de Saúde Pública, 2011; 45(1): 249-257.

5. COLET CR, et al. Uso de plantas medicinais por usuários do serviço público de saúde do município de ljuí/RS. Revista Brasileira de Medicina de Família e Comunidade, 2015; 10(36): 1-13.

6. FERREIRA LLC, et al. Incentivo governamental para Arranjos Produtivos Locais de Plantas Medicinais e Fitoterápicos no âmbito do SUS. Revista Fitos, 2017; 126(1): 54-61.

7. FONTENELE RP, et al. Fitoterapia na Atenção Básica: olhares dos gestores e profissionais da Estratégia Saúde da Família de Teresina (PI). Revista Ciência \& Saúde Coletiva, 2013; 18(1): 2385-2394.

8. GRIBNER C, et al. Use of industrialized herbal medicines by patients attended at the basic health units in the County of Pinhais, Paraná, Brazil. Boletín Latinoamericano y del Caribe de Plantas Medicinales y Aromáticas, 2018; 17(3): 238-248.

9. MATTOS G, et al. Plantas medicinais e fitoterápicos na Atenção Primária em Saúde: percepção dos profissionais. Revista Ciência \& Saúde Coletiva, 2018; 23(11): 3735-3744.

10. BRASIL. Ministério da Saúde. Portaria 971: Política Nacional de Práticas Integrativas e Complementares (PNPIC) no Sistema Único de Saúde. DOU seção 1, 2006.

11. OLIVEIRA AFP, et al. Fitoterapia na atenção básica: estudo com profissionais enfermeiros. Journal of Research Fundamental Care Online, 2017; 9(2): 480-487.

12. PALMA JS, et al. Explanatory models of the professional sector in relation to medicinal plants. Journal of Research Fundamental Care Online, 2015; 7(3): 2998-3008.

13. ROSA CD, et al. Representações e intenção de uso da fitoterapia na atenção básica à saúde. Revista Ciência \& Saúde Coletiva, 2011; 16(1): 311-318.

14. SANTOS JAA, et al. Diagnóstico e Educação em Saúde no Uso de Plantas Medicinais: Relato de Experiência. Revista Ciência em Extensão, 2016; 12(4): 183-196.

15. VARELA DSS, AZEVEDO DM. Opinião de Médicos e Enfermeiros sobre o Uso da Fitoterapia e Plantas Medicinais na Atenção Básica. Revista de Atenção Primária em Saúde, 2014; 17(2): 204-213.

16. ZENI ALB, et al. Utilização de plantas medicinais como remédio caseiro na Atenção Primária em Blumenau, Santa Catarina, Brasil. Revista Ciência \& Saúde Coletiva, 2017; 22(8): 2703-2712. 